\title{
Intra-individual heteroplasmy in the Gentiana tongolensis plastid genome (Gentianaceae)
}

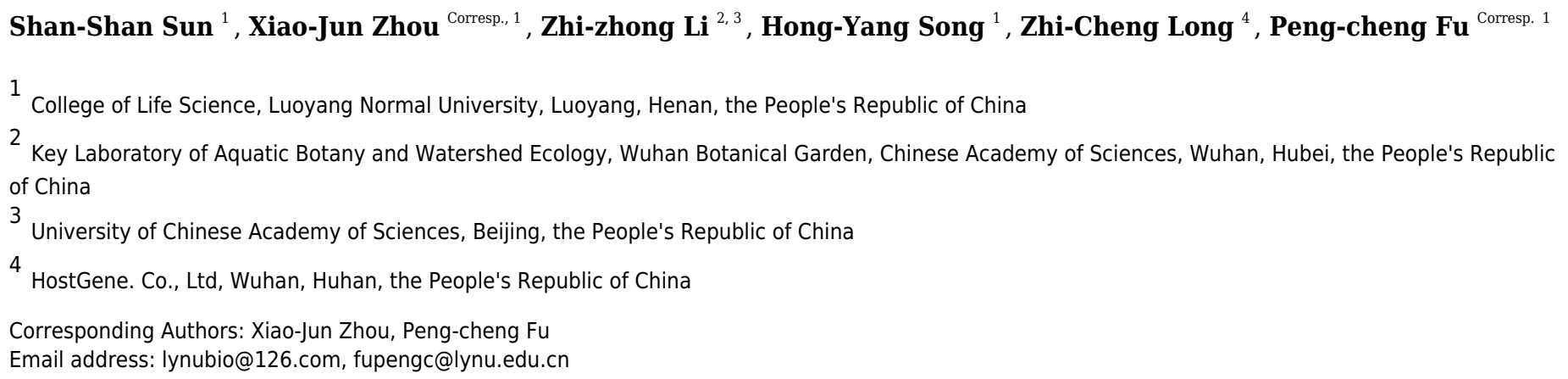

Chloroplasts are typically inherited from the female parent and are haploid in most angiosperms, but rare intra-individual heteroplasmy in plastid genomes has been reported in plants. Here, we report an example of plastome heteroplasmy and its characteristics in Gentiana tongolensis (Gentianaceae). The plastid genome of $G$. tongolensis is 145,757 bp in size and is missing parts of petD gene when compared with other Gentiana species. A total of 112 SNPs and 31 indels with frequencies of more than $2 \%$ were detected in the plastid genome, and most were located in protein coding regions. Most sites with SNP frequencies of more than $10 \%$ were located in six genes in the LSC region. After verification via cloning and Sanger sequencing at three loci, heteroplasmy was identified in different individuals. The cause of heteroplasmy at the nucleotide level in plastome of $G$. tongolensis is unclear from the present data, although biparental plastid inheritance and transfer of plastid DNA seem to be most likely. This study implies that botanists should reconsider the heredity and evolution of chloroplasts and be cautious with using chloroplasts as genetic markers, especially in Gentiana. 


\section{Intra-individual heteroplasmy in the Gentiana 2 tongolensis plastid genome (Gentianaceae)}

3

Shan-Shan Sun ${ }^{1}$, Xiao-Jun Zhou ${ }^{1}$, Zhi-Zhong Li ${ }^{2}, 3$, Hong-Yang Song ${ }^{1}$, Zhi-Cheng Long ${ }^{4}$, PengCheng $\mathrm{Fu}^{1}$

${ }^{1}$ College of Life Science, Luoyang Normal University, Luoyang, Henan, the People's Republic of China

${ }^{2}$ Key Laboratory of Aquatic Botany and Watershed Ecology, Wuhan Botanical Garden, Chinese Academy of Sciences, Wuhan, Hubei, the People's Republic of China ${ }^{3}$ University of Chinese Academy of Sciences, Beijing, the People's Republic of China

${ }^{4}$ HostGene. Co., Ltd, Wuhan, Hubei, the People's Republic of China

Corresponding Author:

Peng-Cheng $\mathrm{Fu}^{1}$

Jiqing Road 6 $6^{\#}$, Luoyang, Henan, 471934, China

Email address: fupengc@lynu.edu.cn

Xiao-Jun Zhou ${ }^{1}$

Jiqing Road 6", Luoyang, Henan, 471934, China

Email address: lynubio@126.com

\section{Abstract}

Chloroplasts are typically inherited from the female parent and are haploid in most angiosperms, but rare intra-individual heteroplasmy in plastid genomes has been reported in plants. Here, we report an example of plastome heteroplasmy and its characteristics in Gentiana tongolensis (Gentianaceae). The plastid genome of $G$. tongolensis is $145,757 \mathrm{bp}$ in size and is missing parts of petD gene when compared with other Gentiana species. A total of 112 SNPs and 31 indels with frequencies of more than $2 \%$ were detected in the plastid genome, and most were located in protein coding regions. Most sites with SNP frequencies of more than $10 \%$ were located in six genes in the LSC region. After verification via cloning and Sanger sequencing at three regions, heteroplasmy was identified in different individuals. The cause of heteroplasmy at the nucleotide level in plastomes of $G$. tongolensis is unclear from the present data, although biparental plastid inheritance and transfer of plastid DNA seem to be most likely. This study implies that botanists should reconsider the heredity and evolution of chloroplasts and be cautious with using chloroplasts as genetic markers, especially in Gentiana. 
39

40

41

42

43

44

45

46

47

48

49

50

51

52

53

54

55

56

57

58

59

60

61

62

63

64

65

66

67

68

69

70

71

72

73

74

75

76

77

78

\section{Introduction}

Chloroplasts transform solar energy into chemical energy stored in the form of carbohydrates, supporting life on Earth. In angiosperms, plastomes are generally highly conserved with a quadripartite structure that contains a pair of inverted repeats (IRs) that separate the remaining regions into one small single-copy region (SSC) and one large single-copy region (LSC) (Palmer, 1985; Jansen et al., 2005). Plastomes range in size from 120 to $160 \mathrm{~kb}$ and contain conserved gene content and order, usually 110-130 genes in angiosperms (Palmer, 1985; Ruhlman \& Jansen, 2014). Most flowering plants exhibit maternal plastid inheritance, and rarely paternal plastid inheritance (Schumann \& Hancock, 1989), but some genera display biparental plastid inheritance to some degree (Mogensen, 1996). At the level of population or species, plastids exhibit sequence and structural polymorphisms (e.g. Sun et al., 2018), but heteroplasmy, the condition of cells having more than one organelle haplotype, has also been reported in limited taxa (Fitter et al., 1996; Garcia, Nicholson \& Nickrent, 2004; Frey, Frey \& Forcioli, 2005; McCauley et al., 2007).

Chloroplast data are ubiquitous in plant systematics research due to its uniparental inheritance, haploid nature, highly conserved structure, and slower evolutionary rate of change compared to nuclear genomes (Wolfe, Li \& Sharp, 1987; Shaw et al., 2014). Therefore, the plastome has been widely applied to reassess classifications, divergence dating, mutational hotspot identification, and genome evolution (e.g. Nikiforova et al., 2013; Yao et al., 2019). The National Center for Biotechnology Information (NCBI) contains more than 7,000 records of complete chloroplast genomes in plants as of April 24 $4^{\text {th }}, 2019$ (title $=$ chloroplast AND complete genome).

As the largest genus in family Gentianaceae, Gentiana contains 15 sections and 362 species (Ho $\&$ Liu, 2001). Gentiana is predominantly alpine around the world, and some taxa have already been cultured (Rybczyński, Davey \& Mikuła, 2015) due to chemical and horticultural value (Ho \& Liu, 2001). The mountain ranges surrounding the Qinghai-Tibetan Plateau (QTP), where roughly 250 species exist (Ho \& Pringle, 1995), are the main diversity center of Gentiana (Favre et al., 2016). Presently, the plastomes of twelve Gentiana species have been sequenced (Fu et al., 2016; Ni et al., 2016; Wang et al., 2017; Sun et al., 2018), which belong to two sections, Cruciata Gaudin and Kudoa (Masamune) Satake \& Toyokuni ex Toyokuni. In addition to these plastomes, a number of plastid fragments have been sequenced and used to reconstruct phylogenetic relationships in Gentiana and Gentianaceae (e.g. Favre et al., 2016). All previous studies tacitly accepted the maternal inheritance and haploid nature of plastomes in these species, and there are no reports regarding heteroplasmy in Gentianaceae.

As part of our ongoing study of Gentiana plastomes, some surprising heteroplasmy was uncovered in G. tongolensis Franchet, which is an annual herb belonging to section Microsperma T. N. Ho serious Suborbisepalae Marquand (Ho \& Liu, 2001). This species is endemic to China with its distribution limited to the southeast of the QTP (Ho \& Liu, 2001). Gentiana tongolensis has been a poorly studied species within a large genus, except for its classification and taxonomy (e.g. Favre et al., 2016). Here, we report heteroplasmy in the plastome of G. tongolensis at the nucleotide level. This information will help botanists to reconsider the heredity and evolution of chloroplasts and to take caution with their use as genetic markers. 


\section{Materials \& Methods}

\section{Sample collection, genome sequencing and assembly}

81 Five individuals of $G$. tongolensis were sampled at Daocheng, Sichuan Province in the QTP $82\left(28^{\circ} 52^{\prime} \mathrm{E}, 100^{\circ} 16^{\prime} \mathrm{N}\right)$. The species was identified by Dr. Peng-Cheng Fu, and its voucher 83 specimens (No. Fu2016162) were deposited in the herbarium of the College of Life Science, 84 Luoyang Normal University. Each sample was collected from a single plant and fast dried by silica 85 gel. One sample (Fu2016162.05) was used for genome sequencing in this study. We performed 86 total genomic DNA isolation, DNA fragmentation, and sequencing library construction following 87 the process described by Fu et al. (2016). The extracted total genomic DNA was quantified by a 88 Qubit3.0 fluorometer (Thermo Fisher Scientific, Waltham, USA) and 1\% agarose electrophoresis. 89 The fragmented genomic DNA was sequenced using the Illumina HiSeq 4000 platform 90 (Novogene, Tianjing, China) yielding 150-bp paired-end reads from a library of around 300-bp 91 DNA fragments. Based on the genome size (Mishiba et al., 2009) and previous plastid genome 92 studies (Fu et al., 2016; Sun et al., 2018) in Gentiana, we obtained approximately 7 Gb of raw data that was deposited in the Genome Sequence Archive of the BIG Data Center (accession no. CRA001588). The raw reads were processed in a relatively standard manner in Trimmomatic 0.38 (Bolger, Lohse \& Usadel, 2014), beginning by trimming and removing bases with qualities less than 20 at both 5' and 3' ends, and reads with less than $36 \mathrm{bp}$ and average base quality less than 15 in a 4-base window. The plastome was de novo assembled in NOVOPlasty 2.6.1 (Dierckxsens, Mardulyn \& Smits, 2016). The plastome sequence of G. tongolensis was deposited in GenBank (accession no. MK251985).

100

101

102

103

104

105

106

107

108

109

110

111

112

113

114

115

116

117

118

\section{Genome annotation and comparative analysis}

The protein coding genes, rRNAs, and tRNAs in the plastome were predicted and annotated using Dual Organellar GenoMe Annotator (Wyman, Janse \& Boore, 2004) and GeSeq (Tillich et al., 2017). Comparative analysis was performed with three Gentiana species that represent the different groups reported (Sun et al., 2018). Plastomes were downloaded from NCBI (G. straminea NC_027441, G. lawrencei var. farreri KX096882, G. stipitata NC_037984), and genome comparisons were performed to identify the differences among taxa using Geneious Basic 5.6.4 (Kearse et al., 2012) and mVISTA (Frazer et al., 2004).

\section{SNP calling and verification}

Reads used for de novo assembly were aligned to the assembled plastome using BWA-MEM (Li, 2013) with default parameters. SNPs or indels were then called using Pisces 5.2.11 (Dunn et al., 2018) with default parameters. Pisces is an Illumina variant caller for calling low frequency variants in tumors by producing a Q-score indicating the confidence that the variant is indeed present. SNPs or indels with variant frequency less than $2 \%$ were omitted to avoid sequencing errors, whose rate can approach 1-2\% in some situations (Schirmer et al. 2016). For reducing the effect of low-quality bases, SNPs with a Q-score of 100 were chosen for downstream analysis.

For verifying the SNPs in the plastome, three regions (GTcpSNP-3, GTcpSNP-4 and GTcpSNP5) located in the LSC with high SNP frequencies were chosen (Fig. 1). GTcpSNP-3, located at the IRa-LSC boundary and ranged from 145,647 to 664 in the plastome, includes parts of $r p s 19$, all of 
$119 \operatorname{trnH}$, and parts of $p s b A$. GTcpSNP-4, ranging from 23,015 to 23,631 in the plastome, is part of 120 gene rров. GTcpSNP-5, ranging from 68,312 to 68,882 in the plastome, is part of gene psbB. 121 Another two regions (GTcpSNP-1 and GTcpSNP-2) with no SNPs were also chosen as controls 122 (Fig. 1). GTcpSNP-1 is located in the SSC region and ranges from 114,570 to 115,206 in the 123 plastome. GTcpSNP-2 is located in the IR region and ranges from 129,395 to 129,965 in the 124 plastome. Verification was performed first in the same individual that was sequenced to obtain the 125 Illumina data and then four more individuals in the same population. Since primers could not be 126 designed for the loci for TaqMan, the method of transformed cloning was adopted. Primers were

127

128

129

130

131

132

133

134

135

136

137

138

139

140

141

142

143

144

145

146

147

148

149

150

151

152

153

154

155

156

157

158 designed using PRIMER version 5.0 (Clarke and Gorley, 2001), and their information is presented in Table S1. PCR was performed in $25-\mathrm{mL}$ volumes containing $20 \mathrm{ng}$ of template DNA, $1 \times$ PCR Buffer, $1.5 \mathrm{mM} \mathrm{MgCl}_{2}, 0.25 \mathrm{mM}$ of each dNTP, $0.3 \mathrm{mM}$ of each primer, and 1 unit of Taq DNA polymerase (Takara, Dalian, China). The PCR cycling profile included an initial step of $5 \mathrm{~min}$ at $95^{\circ} \mathrm{C}$, followed by 35 cycles of denaturation at $95^{\circ} \mathrm{C}$ for $50 \mathrm{~s}, 50 \mathrm{~s}$ of annealing at $54^{\circ} \mathrm{C}$, and $30 \mathrm{~s}$ at $72^{\circ} \mathrm{C}$, and a final extension at $72^{\circ} \mathrm{C}$ for $7 \mathrm{~min}$. PCR products were purified by an eZNA DNA Gel Extraction Kit (Omega Bio-Tek, Guangzhou, China). The concentration of purified PCR products was measured by a NanoDrop 2000c Spectrophotometer (Thermo Scientific), and then the PCR products were ligated into a pMD19-T vector (Takara, Dalian, China) and transformed into Trans $5 \alpha$ Chemically Competent Cells (TransGen, Beijing, China). Positive clones were tested in a 20- $\mu \mathrm{L}$ PCR reaction volume containing 10-100 ng template DNA, $1 \times$ PCR Buffer, $1.5 \mathrm{mM}$ $\mathrm{MgCl}_{2}, 0.2 \mathrm{mM}$ of each dNTP, $0.2 \mathrm{mM}$ of M13F/R, and 1 unit of Taq DNA polymerase (Takara, Dalian, China). PCR was performed under the following program: an initial step of $5 \mathrm{~min}$ at $95^{\circ} \mathrm{C}$ followed by 20 cycles of $30 \mathrm{~s}$ at $95^{\circ} \mathrm{C}, 1 \mathrm{~min}$ at $53^{\circ} \mathrm{C}$, and $30 \mathrm{~s}$ at $72^{\circ} \mathrm{C}$, followed by a final extension step at $72^{\circ} \mathrm{C}$ for $7 \mathrm{~min}$. The positive clones were sequenced using the Sanger method with M13 universal primer.

\section{Genetic analysis of verified loci}

Sequences were aligned with Geneious Alignment and edited in Geneious Basic 5.6.4 (Kearse et al., 2012). Haplotypes were identified in DnaSP 5.1 (Librado \& Rozas, 2009) and deposited in GenBank (nos. MK251953-MK251984). Phylogenetic relationships among haplotypes were reconstructed in PhyML 3.0 (Guindon and Gascuel, 2003) with the GTR model estimated in jModelTest 2.1.7 (Darriba et al., 2012).

\section{Results}

\section{Quality of reads and features of the plastome}

There were 218 million clean reads counting for 18.3 billion bases from high-throughput sequencing. The Q20 and Q30 were 99.99\% and 96.69\%, respectively. The size of the plastid genome was $145,757 \mathrm{bp}$ in length (Table 1). Its circular-mapping assembly possessed the typical quadripartite structure (Shinozaki et al., 1986; Fig. 1) and comprised a pair of IR regions (IRa and $\mathrm{IRb}$ ), one LSC region, and one SSC region, which were 25,359 bp, 78,289 bp, and 16,750 bp, respectively. A comparison of plastomes in Gentiana showed that G. tongolensis has a similar genome structure and gene order with G. straminea and G. stipitata (Fu et al., 2016; Ni et al., 2016; Sun et al., 2018), but had partial gene loss in petD (Fig. S1). 
159

160

161

162

163

164

165

166

167

168

169

170

171

172

173

174

175

176

177

178

179

180

181

182

183

184

185

186

187

188

189

190

191

192

193

194

195

196

197

198

\section{Character of SNPs}

After filtering with variant frequency and Q-score, a total of 112 SNPs and 31 indels were identified in the plastome of $G$. tongolensis. The average sequencing depth of each base at a variant position was 2699x. Among the variant positions, 45 (31.46\%) had a frequency more than 10\%, and all were located in the LSC region. A total of 22 SNPs or indels were detected in the SSC region, and none in the IR regions. The top 20 SNPs or indels with highest frequencies are presented in Table 2. After locating the 143 sites and function annotation, 96 were located in genes, including three in introns, and 47 were located in the intergenic space (Fig. 1; Table S2). Fortyfour out of 45 positions whose SNP frequencies were more than $10 \%$ were located in genes atp $A$, $p s b B, r p o B, r p o C 1, t r n H, p s b C, r p o A$ and $p s a A$. There were $22 \mathrm{C} \rightarrow \mathrm{T}$ plus $15 \mathrm{G} \rightarrow \mathrm{A}$ transitions, and $16 \mathrm{~T} \rightarrow \mathrm{C}$ plus $23 \mathrm{~A} \rightarrow \mathrm{G}$ transitions in the plastome of $G$. tongolensis, and thus no excess of mutations was attributable to $5^{\mathrm{m} C}$ deamination which is a clue as to whether the sequence is nucleus-derived or plastid-derived (Huang et al., 2005).

\section{Verification and genetic characteristics of SNPs}

To verify the polymorphisms detected in the Illumina data, we selected three regions that spanned multiple polymorphisms and two control regions without polymorphisms for cloning and Sanger sequencing (Fig. 1). Verification was first performed for the one individual for Illumina sequencing. In the two regions used as positive controls, 10 and 12 clones were sequenced successfully in GTcpSNP-1 and GTcpSNP-2, respectively, and did not show any SNPs. In GTcpSNP-3, a total of 22 clones were successfully sequenced, and nine SNPs and one indel were detected (Table S3). In GTcpSNP-4, 23 clones were sequenced, and three SNPs were detected (Table S4). In GTcpSNP-5, 26 clones were sequenced, and five indels and two SNPs were detected (Table S5). Focusing on the individual for Illumina sequencing, only two sites (a SNP, position 23411; a indel, position 68770) in the sequences obtained from verification were matched with the sites in the plastome on which our primers were designed (Table 3 ). On the other hand, lots of new variable sites were detected through verification.

For further clarifying the SNPs in the plastome, four more individuals were verified and analyzed with the one individual for Illumina sequencing. For GTcpSNP-1 and GTcpSNP-2, 10 and seven clones were sequenced in two individuals. For GTcpSNP-1 and GTcpSNP-2, a total of 20 sequences with $636 \mathrm{bp}$ and 19 sequences with $571 \mathrm{bp}$ were obtained from three individuals, respectively. No SNP was detected in the two positive control regions in all verified individuals. In GTcpSNP-3 and GTcpSNP-4, a total of 25 and 29 clones were sequenced in two and four individuals, respectively. In GTcpSNP-3, there were 47 obtained sequences ranging from 775-778 bp in length. Nucleotide variations were detected at 39 sites, with 35 base substitutions and four indels, and a total of 22 haplotypes were identified in three individuals (Table S3). In GTcpSNP4, 52 obtained sequences were $614-615$ bp in length. Nucleotide variations were detected at 21 sites, with 19 base substitutions and two indels, and 15 haplotypes were identified in five individuals (Table S4). In GTcpSNP-5, there were 26 obtained sequences ranging from 562-572 bp in length. Nucleotide variations were detected at seven sites which included five indels and two SNPs, and a total of eight haplotypes were identified in one individual (Table S5). Besides the 
199

200

201

202

203

204

205

206

207

208

209

210

211

212

213

214

215

216

217

218

219

220

221

222

223

224

225

226

227

228

229

230

231

232

233

234

235

236

237

238

same sequences with the reference plastome were shared by different individuals, no haplotype was shared among individuals, indicating high heterozygosity in the plastome. We attempted to determine the phylogenetic relationship among haplotypes, but we found no phylogenetic structure among them (results not shown).

\section{Discussion}

Polymorphisms are common in plastid genomes at the level of population or species, but intraindividual heteroplasmy has rarely been reported (Medicago sativa, Fitter et al., 1996; Cynomorium coccineum, Garcia, Nicholson \& Nickrent, 2004; Senecio vulgaris, Frey, Frey \& Forcioli, 2005; Silene vulgaris, McCauley et al., 2007). Due to the modular nature of plant growth, perennial plants can develop the same type of organs independently and at different times during their lifetime, thus leading the intra-individual genomic variation, for example in apples (Gillian et al., 2017); however, this does not explain the heteroplasmy in G. tongolensis, which is an annual herb. Another possibility of the heteroplasmy is due to DNA damage - either DNA damage due to technical reasons (e.g. sample storage) or to biological reasons. DNA damage during sample storage with silica gel maybe one potential artificial factor leading to the appearance of SNPs in Illumina read data. However, the agarose electrophoresis before library construction did not show any signs of degradation. Besides, DNA damage that occurred due to biological processes would lead to random mutations whose frequencies should be very low. However, our data showed that SNPs were mainly located in genes in the LSC region, and the frequencies of a number of SNPs were high. On the other hand, compromise in plastid DNA repair is a potential reason that cannot be excluded by our present data. Because the rate with which we have detected variants among clones within a verified individual was well above expected somatic mutation levels, it seems unlikely that this is a cause for the heteroplasmy in G. tongolensis.

The transfer of plastid DNA, ranging from individual genes (eg. rpl22, Jansen et al., 2010; rpl32, Park, Jansen \& Park, 2015) to as much as $131 \mathrm{~kb}$ in rice (Noutsos, Richly \& Leister, 2005), is an ongoing and ubiquitous process in plants (Martin et al., 1998; Huang et al., 2005). Plastid DNA insertions in the nucleus are in a new mutational environment and have different evolutionary fates compared with plastid genomes (Huang et al., 2005; Noutsos, Richly \& Leister, 2005; Michalovov, Vyskot \& Kejnovsky E. 2013). Chloroplast genomes exhibit very low levels of cytosine methylation (Ayliffe et al., 1998), which is extensive in plant nuclear genomes (Finnegan et al., 1998). Therefore, an excess of mutations attributable to $5^{\mathrm{m}} \mathrm{C}$ deamination, detected by $\mathrm{C} \rightarrow \mathrm{T}$ plus $\mathrm{G} \rightarrow \mathrm{A}$ transitions vs. $\mathrm{T} \rightarrow \mathrm{C}$ plus $\mathrm{A} \rightarrow \mathrm{G}$ transitions, is a clue as to whether the sequence is nucleusderived or plastid-derived (Huang et al., 2005). Because no excess of mutations attributable to $5^{\mathrm{m}} \mathrm{C}$ deamination were observed in this study, it seems that we may not attribute the individual mutations to the transfer of plastid DNA to the nucleus in G. tongolensis. However, more evidence is necessary to uncover the reason for the heteroplasmy.

These findings raise fundamental questions about chloroplast inheritance in Gentiana. Among the seed plants, chloroplasts can be inherited strictly from the female parent, strictly from the male parent, or biparentally (Mogensen, 1996). Most flowering plants exhibit maternal plastid inheritance, but there is rare paternal plastid inheritance among such plants as Medicago 
239 (Schumann \& Hancock, 1989) and Arabidopsis (Azhagiri \& Maliga, 2007). However, some genera 240 display biparental plastid inheritance such as in Coreopsis grandiflora (Mason, Holsinger \& 241 Jansen, 1994), Chamaecyparis obtusa (Shiraishi et al., 2001), Medicago truncatula (Matsushima 242 et al., 2008), and Caprifoliaceae (Hu, Zhang \& Rao, 2008). Paternal, maternal, and biparental 243 plastid inheritance have even been detected from both interspecific and intraspecific crosses in 244 Passiflora (Hansen et al., 2007). Biparental plastid inheritance is a reasonably widespread trait 245 (20\% to nearly one-third) in angiosperms (Smith, 1989; Zhang \& Sodmergen, 2010), and it is 246 probable that heteroplasmy occurs on a limited scale in most groups of angiosperms (Azhagiri \& 247 Maliga, 2007; Hansen et al., 2007). Therefore, this suggests that intra-individual heterozygosity in the $G$. tongolensis plastome may be observed in functionally paralogous copies within an individual caused by biparental plastid inheritance. Consistent with this idea is the fact that all

250

251

252

253

254

255

256

257

258

259

260

261

262

263

264

265

266

267

268

269

270

271

272

273

274

275

276

277

278 high-frequency SNPs detected in the plastome of G. tongolensis were located in coding regions rather than intergenic regions.

Although the plastome is conserved in flowering plants, structural variations such as gene loss, inverted repeats, and rearrangements are common (Downie \& Jansen, 2015; Ruhlman and Jansen, 2018). Recent studies in Gentiana show that the plastome structure is conserved, except for the $n d h$ gene being lost in section Kudoa (Fu et al., 2016; Sun et al., 2018). In the plastome of $G$. tongolensis, we detected partial loss of petD, which encodes subunit IV of the cytochrome $b_{6} / f$ complex. We also found that the $p s b B$, trn $H$ and $r p o B$ genes have distinctly high frequencies of SNPs within an individual in this study. Intra-genomic synonymous rate variations resulting from localized variations in mutation rate can lead to the distribution of SNPs among the coding region (Zhu et al., 2014). However, psbB, trnH and rpoB were not mutational hotspots in Gentiana (Fig. S1; Sun et al., 2018). Although locus-specific substitution rates are not based on gene or protein function (Zhu et al., 2014), the possibility that the three genes may have experienced or are experiencing unusual evolutionary processes cannot be excluded. Serious mutations in the plastid genome may cause nuclear-plastid incompatibility, which is the most important driving force for altering modes of organelle inheritance (Hurst et al., 1996). One hypothesis suggested is that biparental plastid inheritance may have been derived to rescue angiosperms with nuclear-plastid incompatibility caused by defective plastids (Zhang \& Sodmergen, 2010). The case of $G$. tongolensis may support this hypothesis, but more evidence is required.

Plastid DNA is a ubiquitous molecular marker in plant systematics and population genetics research, such as DNA barcoding (Group, 2009) and plant phylogeography (Morris \& Shaw, 2018), because of its uniparental inheritance and haploid nature in flowering plants (Wolfe, Li \& Sharp, 1987; Shaw et al., 2014). In most angiosperms, plastids are under maternal inheritance, thus plastid datasets can provide information on past changes in species distribution when the colonization of new habitats occurs through seeds (Petit et al., 2003). They also offer insight into plant phylogenetic relationships by eliminating complex evolutionary events such as hybridization or introgression. Plastomes have also been widely applied for reassessing classifications, divergence dating, and mutational hotspot identification (eg. Nikiforova et al., 2013; Yao et al., 2019). Biparental inheritance of plastids will result in divergent plastids within an individual and 
279

280

281

282

283

284

285

286

287

288

289

290

291

292

293

294

295

296

297

298

299

300

301

302

303

304

305

306

307

308

309

310

311

312

313

314

315

316

317

318

are functionally paralogous (Wolfe \& Randle, 2004), thus random sampling of nucleotide sequences from different plastid haplotypes or from paralogous sequences may affect the resulting gene tree topology and the resulting inferences (Wolfe \& Randle, 2004; Hansen et al., 2007). Examples have been presented in the phylogenetic inferences in Passiflora (Hansen et al., 2007) and Picea (Sullivan et al., 2017). Since we detected intra-individual plastid genome heterozygosity in $G$. tongolensis, the chloroplast phylogeny may be problematic for phylogenetic inference and not accurately represent ancestor-descendant relationships, especially in Gentiana. Besides phylogenetic studies, intra-individual chloroplast heterozygosity can also affect population studies that are sometimes based on the inference of genetic diversity within and between populations. Therefore, before chloroplast heteroplasmy is further studied in more taxa, additional attention should be paid when chloroplast sequences are used.

\section{Conclusions}

In summary, we reported a case of heteroplasmy and its characteristics in the G. tongolensis plastome, but the underlying cause of it is unclear from the present data. Biparental plastid inheritance and transfer of plastid DNA seemed to be most likely, and to better address this question, it will be necessary to collect more data from this species. Hopefully, this study will encourage botanists to reconsider the heredity and evolution of chloroplasts and exercise caution when using them as genetic markers, especially in Gentiana.

\section{Acknowledgements}

We thank the three anonymous reviewers and the editor for their helpful comments and suggestions on the manuscript.

\section{References}

Ayliffe MA, Scott NS, Timmis JN. 1998. Analysis of plastid DNA-like sequences within the nuclear genomes of higher plants. Molecular Biology and Evolution 15: 738-745 DOI 10.1093/oxfordjournals.molbev.a025977.

Azhagiri AK, Maliga P. 2007. Exceptional paternal inheritance of plastids in Arabidopsis suggests that low-frequency leakage of plastids via pollen may be universal in plants. The Plant Journal 52: 817-823 DOI 10.1111/j.1365-313X.2007.03278.x.

Birky Jr CW. 2001. The inheritance of genes in mitochondria and chloroplasts: laws, mechanisms, and models. Annual Review of Genetics 35: 125-148 DOI 10.1146/annurev.genet.35.102401.090231.

Bolger AM, Lohse M, Usadel B. 2014. Trimmomatic: a flexible trimmer for Illumina sequence data. Bioinformatics 30: 2114-2120 DOI 10.1093/bioinformatics/btu170.

Clarke KR, Gorley RN. 2001. PRIMER v5: User Manual / Tutorial. PRIMER-E Ltd, 99 Plymouth, p 91.

Darriba D, Taboada GL, Doallo R, Posada D. 2012. jModelTest 2: more models, new heuristics and parallel computing. Nature Methods 9: 772 DOI 10.1038/nmeth.2109.

Dierckxsens N, Mardulyn P, Smits G. 2016. NOVOPlasty: de novo assembly of organelle genomes from whole genome data. Nucleic Acids Research 45: e18-e18 DOI 10.1093/nar/gkw955. 
319

320

321

322

323

324

325

326

327

328

329

330

331

332

333

334

335

336

337

338

339

340

341

342

343

344

345

346

347

348

349

350

351

352

353

354

355

356

357

358

Downie SR, Jansen RK. 2015. A comparative analysis of whole plastid genomes from the Apiales: expansion and contraction of the inverted repeat, mitochondrial to plastid transfer of DNA, and identification of highly divergent noncoding regions. Systematic Botany 40: 336-351 DOI 10.1600/036364415X686620.

Dunn T, Berry G, Emig-Agius D, Jiang Y, Lei S, Iyer A, Udar N, Chuang HY, Hegarty J, Dickover M, Klotzle B, Robbins J, Bibikova M, Peeters M, Stromberg M. 2018. Pisces: An accurate and versatile variant caller for somatic and germline next-generation sequencing data. BioRxiv, 291641 DOI 10.1101/291641.

Esposito D, Higgs DC, Drager RG, Stern DB, Girard-Bascou J. 2001. A nucleus-encoded suppressor defines a new factor which can promote petD mRNA stability in the chloroplast of Chlamydomonas reinhardtii. Current Genetics 39: 40-48 DOI 10.1007/s002940000179.

Favre A, Michalak I, Chen CH, Wang JC, Pringle JS, Matuszak S, Sun H, Yuan YM, Struwe L, Muellner-Riehl AN. 2016. Out-of-Tibet: the spatio-temporal evolution of Gentiana (Gentianaceae). Journal of Biogeography 43: 1967-1978 DOI 10.1111/jbi.12840.

Finnegan EJ, Genger RK, Peacock WJ, Dennis ES. 1998. DNA methylation in plants. Annual Review of Plant Biology 49: 223-247 DOI 10.1146/annurev.arplant.49.1.223

Fitter JT, Thomas MR, Rose RJ, Steelescott N. 1996. Heteroplasmy of the chloroplast genome of Medicago sativa L. cv 'Regen S' confirmed by sequence analysis. Theoretical and Applied Genetics 93: 685-690 DOI 10.1007/BF00224063.

Frazer KA, Pachter L, Poliakov A, Rubin EM, Dubchak I. 2004. VISTA: computational tools for comparative genomics. Nucleic Acids Research 32: W273-W279 DOI 10.1093/nar/gkh458.

Frey JE, Frey B, Forcioli D. 2005. Quantitative assessment of heteroplasmy levels in Senecio vulgaris chloroplast DNA. Genetica 123: 255-261 DOI 10.1007/s10709-004-3711-y.

Fu PC, Zhang YZ, Geng HM, Chen SL. 2016. The complete chloroplast genome sequence of Gentiana lawrencei var. farreri (Gentianaceae) and comparative analysis with its congeneric species. PeerJ 4: e2540 DOI 10.7717/peerj.2540.

Garcia MA, Nicholson EH, Nickrent DL. 2004. Extensive intra-individual variation in plastid rDNA sequences from the holoparasite Cynomorium coccineum (Cynomoriaceae). Journal of Molecular Evolution 58: 322-332 DOI 10.1007/s00239-003-2554-y.

Gillian R, Eli A, Salomon TC, Jim C. 2017. Intraindividual genomic variation and mutation in apple (Malus $\times$ domestica). Abstracts of Botany 2017, Texas.

Group CPW. 2009. A DNA barcode for land plants. Proceedings of the National Academy of Sciences 106: 12794-12797 DOI 10.1073/pnas.0905845106.

Guindon S, Gascuel O. 2003. A simple, fast and accurate method to estimate large phylogenies by maximum-likelihood. Systematic Biology. 52: 696-704 DOI 10.1080/10635150390235520.

Hansen AK, Escobar LK, Gilbert LE, Jansen RK. 2007. Paternal, maternal, and biparental inheritance of the chloroplast genome in Passiflora (Passifloraceae): implications for phylogenetic studies. American Journal of Botany 94: 42-46 DOI 10.3732/ajb.94.1.42. 
359

360

361

362

363

364

365

366

367

368

369

370

371

372

373

374

375

376

377

378

379

380

381

382

383

384

385

386

387

388

389

390

391

392

393

394

395

396

397

398

Hu Y, Zhang Q, Rao G. 2008. Occurrence of plastids in the sperm cells of Caprifoliaceae: biparental plastid inheritance in angiosperms is unilaterally derived from maternal inheritance. Plant and Cell Physiology 49: 958-968 DOI 10.1093/pcp/pcn069.

Huang CY, Grünheit N, Ahmadinejad N, Timmis JN, Martin W. 2005. Mutational decay and age of chloroplast and mitochondrial genomes transferred recently to angiosperm nuclear chromosomes. Plant Physiology 138: 1723-1733 DOI 10.1104/pp.105.060327.

Ho TN, Pringle JS. 1995. Gentianaceae. In: Wu ZY, Raven PH. eds. Flora of China. Beijing, China: Science Press, 1-140.

Ho TN, Liu SW. 2001. A worldwide monograph of Gentiana. Beijing, China: Science Press.

Jansen RK, Raubeson LA, Boore JL, dePamphilis CW, Chumley TW, Haberle RC, Wyman SK, Alverson AJ. Peery R, Herman SJ, Fourcade HM, Kuehl JV, McNeal JR, Leebens-Mack J, Cui LY. 2005. Methods for obtaining and analyzing chloroplast genome sequences. Methods in Enzymology 395: 348-384 DOI 10.1016/S0076-6879(05)95020-9.

Jansen RK, Saski C, Lee SB, Hansen AK, Daniell H. 2010. Complete plastid genome sequences of three rosids (Castanea, Prunus, Theobroma): evidence for at least two independent transfers of rpl22 to the nucleus. Molecular Biology and Evolution 28: 835847 DOI 10.1093/molbev/msq261.

Kearse M, Moir R, Wilson A, Stones-Havas S, Cheung M, Sturrock S, Buxton S, Cooper A, Markowitz S, Duran C, Thierer T, Ashton B, Meintjes P, Drummond A. 2012. Geneious Basic: an integrated and extendable desktop software platform for the organization and analysis of sequence data. Bioinformatics 28: 1647-1649 DOI 10.1093/bioinformatics/bts199.

Librado P, Rozas J. 2009. DnaSP v5: a software for comprehensive analysis of DNA polymorphism data. Bioinformatics 25: 1451-1452 DOI 10.1093/bioinformatics/btp187.

Li H. 2013. Aligning sequence reads, clone sequences and assembly contigs with BWA-MEM. arXiv preprint arXiv:1303.3997.

Lohse M, Drechsel O, Bock R. 2007. OrganellarGenomeDRAW (OGDRAW): a tool for the easy generation of high-quality custom graphical maps of plastid and mitochondrial genomes. Current Genetics 52: 267-274 DOI 10.1007/s00294-007-0161-y.

Martin W, Stoebe B, Goremykin V, Hansmann S, Hasegawa M, Kowallik KV. 1998. Gene transfer to the nucleus and the evolution of chloroplasts. Nature 393: 162-165 DOI $10.1038 / 30234$.

Mason RJ, Holsinger KE, Jansen RK. 1994. Biparental inheritance of the chloroplast genome in Coreopsis (Asteraceae). Journal of Heredity 85: 171-173 DOI 10.1093/oxfordjournals.jhered.a111430.

Matsushima R, Hu Y, Toyoda K, Sakamoto W. 2008. The model plant Medicago truncatula exhibits biparental plastid inheritance. Plant and Cell Physiology 49: 81-91 DOI $10.1093 / \mathrm{pcp} / \mathrm{pcm} 170$.

McCauley DE, Sundby AK, Bailey MF, Welch ME. 2007. Inheritance of chloroplast DNA is not strictly maternal in Silene vulgaris (Caryophyllaceae): evidence from experimental 
399

400

401

402

403

404

405

406

407

408

409

410

411

412

413

414

415

416

417

418

419

420

421

422

423

424

425

426

427

428

429

430

431

432

433

434

435

436

437

438

crosses and natural populations. American Journal of Botany 94: 1333-1337 DOI

10.3732/ajb.94.8.1333.

Michalovova M, Vyskot B, Kejnovsky E. 2013. Analysis of plastid and mitochondrial DNA insertions in the nucleus (NUPTs and NUMTs) of six plant species: size, relative age and chromosomal localization. Heredity 111: 314-320. DOI 10.1038/hdy.2013.51.

Mishiba KI, Yamane K, Nakatsuka T, Nakano Y, Yamamura S, Abe J, Kawamura H, Takahata Y, Nishihara M. 2009. Genetic relationships in the genus Gentiana based on chloroplast DNA sequence data and nuclear DNA content. Breeding Science 59: 119-127 DOI 10.1270/jsbbs.59.119.

Mogensen HL. 1996. The hows and whys of cytoplasmic inheritance in seed plants. American Journal of Botany 83: 383-404 DOI 10.1002/j.1537-2197.1996.tb12718.x.

Morris AB, Shaw J. 2018. Markers in time and space: A review of the last decade of plant phylogeographic approaches. Molecular Ecology 27: 2317-2333 DOI 10.1111/mec.14695.

Ni L, Zhao Z, Xu H, Chen S, Dorje G. 2016. Chloroplast genome structures in Gentiana (Gentianaceae), based on three medicinal alpine plants used in Tibetan herbal medicine. Current Genetics 63: 241-252 DOI 10.1007/s00294-016-0631-1.

Nikiforova SV, Cavalieri D, Velasco R, Goremykin V. 2013. Phylogenetic analysis of 47 chloroplast genomes clarifies the contribution of wild species to the domesticated apple maternal line. Molecular Biology and Evolution 30: 1751-1760 DOI 10.1093/molbev/mst092.

Noutsos C, Richly E, Leister D. 2005. Generation and evolutionary fate of insertions of organelle DNA in the nuclear genomes of flowering plants. Genome Research 15: 616-628 DOI 10.1101/gr.3788705.

Park S, Jansen RK, Park S. 2015. Complete plastome sequence of Thalictrum coreanum (Ranunculaceae) and transfer of the rpl32 gene to the nucleus in the ancestor of the subfamily Thalictroideae. BMC Plant Biology 15: 40 DOI 10.1186/s12870-015-0432-6.

Palmer JD. 1985. Comparative organization of chloroplast genomes. Annual Review of Genetics 19: 325-354 DOI 10.1146/annurev.ge.19.120185.001545.

Petit RJ, Aguinagalde I, De Beaulieu JL, Bittkau C, Brewer S, Cheddadi R, Ennos R, Fineschi S, Grivet D, Lascoux M, Mohanty A, Müller-Starck G, Demesure-Musch B, Palmé A, Martín JP, Rendell S, Vendramin GG. 2003. Glacial refugia: hotspots but not melting pots of genetic diversity. Science 300: 1563-1565 DOI 10.1126/science.1083264.

Ruhlman TA, Jansen RK. 2014. The plastid genomes of flowering plants. In Ruhlman TA, Jansen RK, Maliga P. eds. Chloroplast Biotechnology. Totowa, USA: Humana Press, 3-38 DOI 10.1007/978-1-62703-995-6_1.

Ruhlman TA, Jansen RK. 2018. Aberration or Analogy? The Atypical Plastomes of Geraniaceae. In Chaw SM, Jansen RK, eds. Advances in Botanical Research. Cambridge, UK: Academic Press, 223-262.

Rybczyński JJ, Davey MR, Mikuła A. 2015. The Gentianaceae-Volume 2: Biotechnology and Applications. Springer. 
439

440

441

442

443

444

445

446

447

448

449

450

451

452

453

454

455

456

457

458

459

460

461

462

463

464

465

466

467

468

469

470

471

472

473

474

475

476

477

478

Schirmer M, D’Amore R, Ijaz UZ, Hall N, Quince C. 2016. Illumina error profiles: resolving fine-scale variation in metagenomic sequencing data. BMC Bioinformatics 17: 125 DOI 10.1186/s12859-016-0976-y.Schumann CM, Hancock JF. 1989. Paternal inheritance of plastids in Medicago Sativa. Theoretical and Applied Genetics 78: 863-866 DOI 10.1007/BF00266672.

Shaw J, Shafer HL, Leonard OR, Kovach MJ, Schorr M, Morris AB. 2014. Chloroplast DNA sequence utility for the lowest phylogenetic and phylogeographic inferences in angiosperms: the tortoise and the hare IV. American Journal of Botany 101: 1987-2004 DOI 10.3732/ajb.1400398.

Shiraishi S, Maeda H, Toda T, Seido K, Sadaki Y. 2001. Incomplete paternal inheritance of chloroplast DNA recognized in Chamaecyparis obtusa using an intraspecific polymorphism of the trnD-trnY intergenic spacer region. Theoretical and Applied Genetics 102: 935-941 DOI 10.1007/s00122-001-0554-0.

Smith SE. 1989. Biparental inheritance of organelles and its implications in crop improvement. Plant Breeding Reviews 6: 361-393 DOI 10.1002/9781118061039.ch9.

Sullivan AR, Schiffthaler B, Thompson SL, Street NR, Wang XR. 2017. Interspecific plastome pecombination reflects ancient reticulate evolution in Picea (Pinaceae). Molecular Biology and Evolution 34: 1689-1701 DOI 10.1093/molbev/msx111.

Sun SS, Fu PC, Zhou XJ, Cheng YW, Zhang FQ, Chen SL, Gao QB. 2018. The complete plastome sequences of seven species in Gentiana sect. Kudoa (Gentianaceae): insights into plastid gene loss and molecular evolution. Frontiers in Plant Science 9: 493 DOI 10.3389/fpls.2018.00493.

Tillich M, Lehwark P, Pellizzer T, Ulbricht-Jones ES, Fischer A, Bock R, Greiner S. 2017. GeSeq-versatile and accurate annotation of organelle genomes. Nucleic Acids Research $\mathbf{4 5}$ : W6-W11 DOI 10.1093/nar/gkx391.

Wang XF, Yang N, Su J, Zhang H, Cao XY. 2017. The complete chloroplast genome of Gentiana macrophylla, Mitochondrial DNA Part B 2: 395-396 DOI 10.1080/23802359.2017.1347831.

Wolfe KH, Li WH, Sharp PM. 1987. Rates of nucleotide substitution vary greatly among plant mitochondrial, chloroplast, and nuclear DNAs. Proceedings of the National Academy of Sciences 84: 9054-9058 DOI 10.1073/pnas.84.24.9054.

Wolfe AD, Randle CP. 2004. Recombination, heteroplasmy, haplotype polymorphism, and paralogy in plastid genes: implications for plant molecular systematics. Systematic Botany 29: 1011-1020 DOI 10.1600/0363644042451008.

Wyman SK, Janse RK, Boore JL. 2004. Automatic annotation of organellar genomes with DOGMA. Bioinformatics 20: 3252-3255 DOI 10.1093/bioinformatics/bth352.

Yao G, Jin J, Li H, Yang J, Mandala VS, Croley M, Mostow R, Douglas NA, Chase MW, Christenhusz M, Soltis DE, Soltis PS, Smith SA, Brockington SE, Moore MJ, Yi TS, Li DZ. 2019. Plastid phylogenomic insights into the evolution of Caryophyllales. Molecular Phylogenetics and Evolution 134: 74-86 DOI 10.1016/j.ympev.2018.12.023. 
479 Zhang Q, Sodmergen. 2010. Why does biparental plastid inheritance revive in angiosperms? 480 Journal of Plant Research 123: 201-206 DOI 10.1007/s10265-009-0291-z.

481 Zhu A, Guo W, Jain K, Mower JP. 2014. Unprecedented heterogeneity in the synonymous 482 substitution rate within a plant genome. Molecular Biology and Evolution 31: 1228-1236 $483 \quad$ DOI $10.1093 / \mathrm{molbev} / \mathrm{msu} 079$.

484 


\section{Table $\mathbf{1}$ (on next page)}

Basic information of the plastome and its SNPs or indels in Gentiana tongolensis. 
1

\begin{tabular}{cccc}
\hline$\square$ & Length (bp) & No. & $\begin{array}{c}\text { Percentage of } \\
\text { polymorphic sites (\%) }\end{array}$ \\
\hline LSC & 78,289 & 121 & 84.62 \\
IR & 25,359 & 0 & 0 \\
SSC & 16,750 & 22 & 15.38 \\
Total & 145,757 & 143 & 100 \\
\hline
\end{tabular}

2 


\section{Table 2 (on next page)}

The 20 SNPs or indels with highest frequencies in the plastome of a single individual of Gentiana tongolensis.

Ref: reference nucleotide; Alt: alternative nucleotide; No.: number of high-quality bases. 
1

\begin{tabular}{|c|c|c|c|c|c|c|}
\hline Position & $\begin{array}{l}\text { Reference } \\
\text { Base }\end{array}$ & SNP & $\begin{array}{l}\text { Depth of } \\
\text { coverage }\end{array}$ & $\begin{array}{l}\text { Frequency } \\
\text { of SNP }\end{array}$ & Location & Region \\
\hline 14 & $\mathrm{~A}$ & $\mathrm{G}$ & 2843 & 0.31 & $\operatorname{trnH}$ & LSC \\
\hline 30 & A & G & 3265 & 0.297 & $\operatorname{trn} H$ & LSC \\
\hline 54 & A & G & 3794 & 0.281 & $\operatorname{trn} H$ & LSC \\
\hline 68516 & CTGTAAA & $\mathrm{C}$ & 4405 & 0.255 & $p s b B$ & LSC \\
\hline 68943 & G & A & 3819 & 0.201 & $p s b B$ & LSC \\
\hline 69026 & $\mathrm{G}$ & A & 3780 & 0.2 & $p s b B$ & LSC \\
\hline 38948 & $\mathrm{~T}$ & $\mathrm{G}$ & 3792 & 0.184 & psaA & LSC \\
\hline 68569 & $\mathrm{~A}$ & G & 3998 & 0.171 & $p s b B$ & LSC \\
\hline 68436 & $\mathrm{C}$ & $\mathrm{T}$ & 3866 & 0.169 & $p s b B$ & LSC \\
\hline 23192 & A & G & 3545 & 0.155 & rрoB & LSC \\
\hline 68769 & GATTGAATT & $\mathrm{G}$ & 3672 & 0.153 & $p s b B$ & LSC \\
\hline 22457 & A & $\mathrm{G}$ & 3518 & 0.149 & rроB & LSC \\
\hline 23411 & $\mathrm{C}$ & $\mathrm{T}$ & 3586 & 0.145 & rроB & LSC \\
\hline 9086 & G & A & 3651 & 0.144 & $\operatorname{atp} A$ & LSC \\
\hline 22052 & $\mathrm{C}$ & $\mathrm{T}$ & 3783 & 0.144 & rроB & LSC \\
\hline 9142 & $\mathrm{C}$ & A & 3700 & 0.14 & $\operatorname{atp} A$ & LSC \\
\hline 9137 & $\mathrm{~T}$ & G & 3701 & 0.139 & $\operatorname{atp} A$ & LSC \\
\hline 32024 & G & A & 3374 & 0.138 & $p s b C$ & LSC \\
\hline 9119 & $\mathrm{~T}$ & $\mathrm{C}$ & 3617 & 0.137 & $\operatorname{atp} A$ & LSC \\
\hline 22169 & $\mathrm{C}$ & $\mathrm{T}$ & 3841 & 0.136 & rpoB & LSC \\
\hline
\end{tabular}




\section{Table 3 (on next page)}

Summary of verified Sanger sequenced data with Illumina sequenced data in a single individual of Gentiana tongolensis. 
2

\begin{tabular}{lcc}
\hline Position in plastome & 23,411 & 68,770 \\
\hline Function & $r p o B$ & $p s b B$ \\
Illumina sequencing data & $\mathrm{C} / \mathrm{T}$ & ATTGAATT/------- \\
$\quad$ Frequency of SNP/indel & 0.145 & 0.153 \\
Sanger sequencing data & $\mathrm{C} / \mathrm{T}$ & ATTGAATT/------- \\
$\quad$ Frequency of SNP/indel & 0.043 & 0.038 \\
\hline
\end{tabular}

3 


\section{Figure 1}

The structural map of Gentiana tongolensis plastome.

Genes drawn inside the circle are transcribed clockwise, and those outside are transcribed counterclockwise. Genes belonging to different functional groups are shown in different colors. Dots represent SNPs or indels detected from Illumina data in a single individual of $G$. tongolensis. Detailed information about SNPs or indels are presented in Table S2. Five regions (from GTcPSNP-1 to GTcPSNP-5) used for verification are indicated by light blue boxes. 


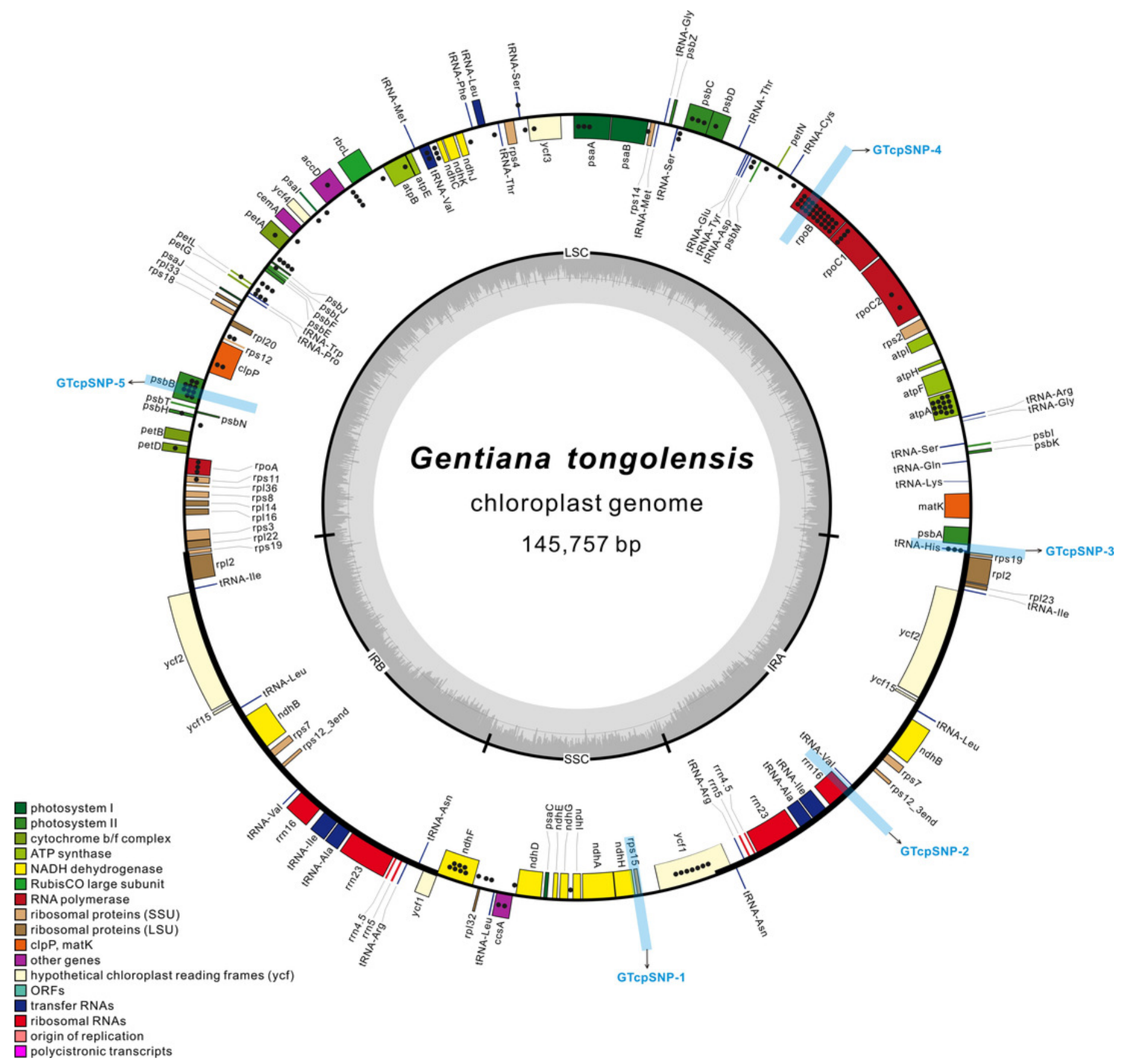

